\title{
Adaptive Detection in Asynchronous Code-Division Multiple-Access System in Multipath Fading Channels
}

\author{
Wen-Sheng Hou and Bor-Sen Chen, Senior Member, IEEE
}

\begin{abstract}
In code-division multiple-access systems transmitting data over time-varying multipath channels, both intersymbol interference (ISI) and multiple-access interference (MAI) arise. In this paper, we address interference suppression, multipath diversity and processing gain protection for multiuser detection with less noise enhancement by using a parallel cancelling scheme. The proposed detector consists of RAKE filter, forward filter, and feedback filter with different functions for each filter. The RAKE filter increases the signal-to-noise ratio by taking the advantage of multipath and code diversities. The forward filter is proposed, in combination with the feedback filter, to remove the effects of MAI and ISI by parallel cancellation. In order to avoid performance deterioration due to unreliable initial estimation in the parallel cancellation, a cost function with proper weighting is introduced to improve the performance of the proposed detector. In the proposed design method, a recursive least square algorithm is employed to update the tap-coefficients of all filters for MAI and ISI cancellation. Finally, the performance of the proposed detector is analyzed and compared with other detectors.
\end{abstract}

Index Terms-CDMA, multipath, fading channels, multiuser detection, parallel interference cancellations.

\section{INTRODUCTION}

I N A NUMBER of different communications applications, a common transmission medium must be shared among many users. Channel resources are traditionally assigned to different users by partitioning the channel into distinct subchannels (either in the time or the frequency domain) and allocating each subchannel to a different user. In such time-division or freqencydivision multiple-access systems, each user transmits during its prespecified time slot (or frequency bin), thereby avoiding any multiple-access interference (MAI) and essentially operating in an equivalent single-channel environment. Unfortunately, this static allocation of resources does not make efficient use of the channel when each user's transmission is infrequent or in bursts. In such cases, a more efficient multiple-access scheme is required, which would dynamically allocate the channel resources depending on the active users' needs.

One approach is to allow all users to transmit at the same time and frequency slot and design the receiver so that it can simul-

Paper approved by B. Aazhang, the Editor for Spread Spectrum Networks of the IEEE Communications Society. Manuscript received February 28, 1998; revised August 13, 1999. This work was supported by the National Science Council under Contract NSC 88-2213-E-007-047.

The authors are with the Department of Electrical Engineering, National Tsing-Hua University, Hsin Chu 300, Taiwan, R.O.C. (e-mail bschen@moti.ee.nthu.edu.tw).

Publisher Item Identifier S 0090-6778(00)04010-1. taneously demodulate more than one user. In direct-sequence code-division multiple-access (DS-CDMA) systems, several independent users share a common channel by modulating a set of signature waveforms. If all mobile radio signals arriving at the base station are synchronized and the assigned signatures are orthogonal, then a bank of decoupled single-user detectors consisting of matched filters followed by threshold detectors [1] will achieve optimal demodulation. However, in most CDMA systems of practical importance, where the transmitter sends information in time-varying multipath channels, both ISI among the data symbols of the same user and MAI among data symbols of different users arise.

CDMA systems using linear and nonlinear interference canceling techniques have shown even better performance if the receiver has knowledge of the spreading codes of all users, received powers of interferers or channel parameters [4], [9], [16]. However, in the asynchronous CDMA transmission system, it is quite difficult to estimate the received powers over a given time interval. Besides, estimation of channel parameters is also not easy, especially for a fast-varying mobile communications channel.

The conventional suboptimum receiver [2] consists of a bank of matched filters where each user detects its own signal without the knowledge of the other users signals. This is often inefficient because MAI is treated as noise and such receivers may require a very tight power control. Several suboptimum detectors have been found to show better performance than the conventional receiver. Minimum mean-square-error (MMSE) and zero-forcing (ZF) equalizers for asynchronous CDMA systems are presented in [4]. In [5], a decorrelating decision-feedback multiuser detector for synchronous CDMA channels is presented. All the detectors mentioned above combat MAI but do not take intersymbol interference (ISI) into account. MMSE linear equalizers for continuous transmission are presented in [6]-[8], which take into account ISI and MAI. In [8], a ZF equalizer for continuous transmission eliminating both ISI and MAI is presented. The disadvantage of a ZF equalizer is that it causes noise enhancement. The MMSE improves the situation by causing less noise enhancement, but results in loss of resistance to the near-far problem.

Another important group of detectors can be classified as subtractive interference cancellation (SIC) detectors and parallel interference cancellation (PIC) detectors. The SIC detector reduces interference by serial cancellation, i.e., subtraction of the user's signals in order to decrease signal strengths [9]-[11]. In contrast, the PIC detector, which estimates and subtracts out 


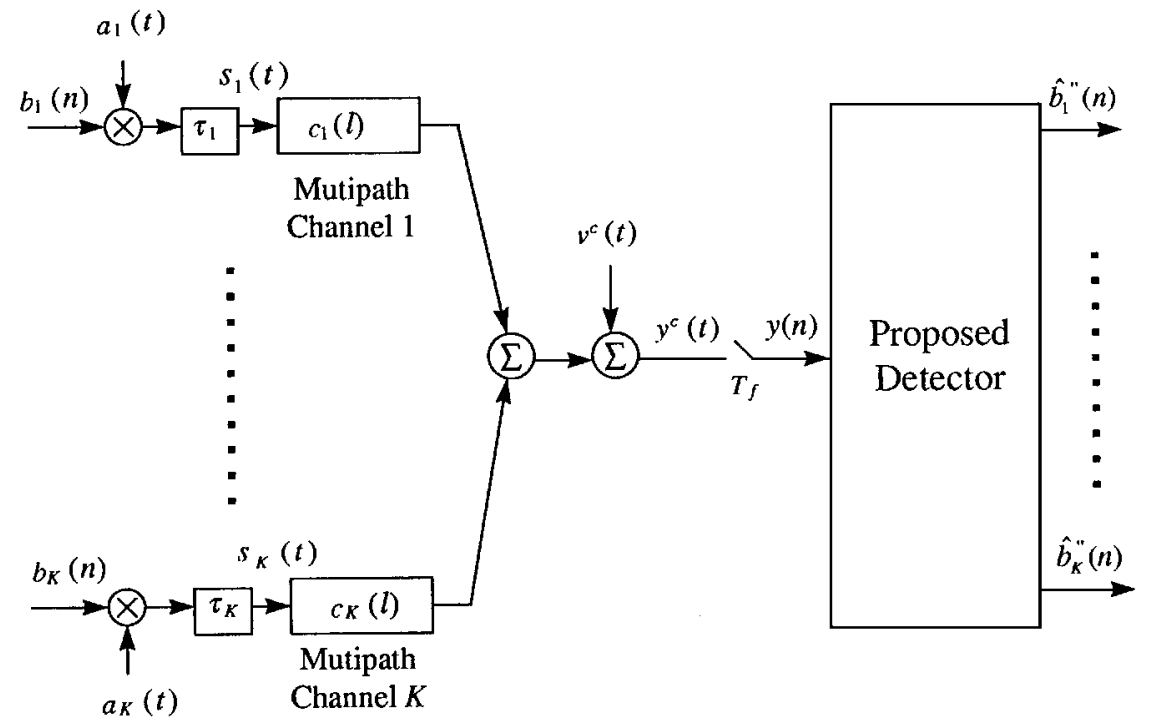

Fig. 1. Block diagram of a CDMA transmission system.

the interference to produce interference-free are considered in [3], [9] and [12]. Joint detection techniques are investigated in [13]-[15]. These detectors are similar to feedback equalizers used to combat ISI. In feedback equalization, detected symbols are fed back in order to cancel part of the ISI. Therefore, these detectors can cancel the MAI without noise enhancement. However, a potential problem with the SIC and PIC detector occurs if the initial symbol estimation is not reliable [19]. In this situation, if the bit estimate is wrong, the interference effect of that bit on the signal-to-noise ratio (SNR) is quadrupled.

This study concentrates on developing an adaptive detector to estimate data sequences simultaneously from all the active users. In the proposed method, the effects of ISI and MAI are reduced and the multipath and code diversities are exploited, with less noise enhancement without a priori channel knowledge. The adaptive detector consists of RAKE filters, forward filters, feedback filters, and threshold decision devices. The RAKE filter exploits multipath diversity, processing gain protection, and suppressing precursor ISI and precursor MAI. The forward filter is developed to cancel the main-cursor MAI, while the feedback filter is developed to cancel the postcursor ISI and postcursor MAI. In order to efficiently exploit the multipath diversity and processing gain and to cancel the MAI and ISI without large noise enhancement, the decision output is used to cancel the ISI and MAI by forward filter and feedback filter. Since the forward filter and feedback filter all work in noise-free environments, both filters cancel ISI and MAI without noise enhancement, and thereby give the RAKE filter great flexibility in handling multipath diversity and processing gain. The proposed adaptive detector estimates and subtracts out all of the maincursor MAI for each user by parallel cancellation. However, a potential deterioration problem with the proposed parallel canceller will occurs if the initial data estimates are not reliable [19]. Therefore, using a cost function with proper weighting as a design criterion significantly improves the performance of the proposed adaptive detector. We can take advantage of this feature of the proposed approach to design an adaptive detector based on the MMSE method, which provides for multipath diversity, processing gain protection, MAI cancellation, and ISI cancellation with less noise enhancement.

From the simulation results, the proposed adaptive detector has been shown to have the ability to remove the effects of MAI and ISI. In addition, the proposed detector provides multipath diversity (including processing gain) protection and near-far resistance.

The remainder of the paper is organized as follows. The system model and problem statement are described in Section II. The proposed adaptive detector algorithm is derived in Section III, and Section IV presents the simulation results. The paper's conclusions are presented in Section V.

\section{SYSTEM DESCRIPTION}

\section{A. Model for Asynchronous CDMA System}

An asynchronous CDMA system is considered in which $K$ users access the same channel at the same time and on the same frequency band. Fig. 1 shows the equivalent baseband representation of the asynchronous CDMA system used in this paper. The transmitted signal of user $k$ is a spread-spectrum (SS) binary phase-shift keying (BPSK) signal, represented as

$$
s_{k}(t)=\sum_{n} b_{k}(n) a_{k}\left(t-n T_{b}-\tau_{k}\right)
$$

where $b_{k}(n)$ is the real-valued transmitted data symbol \pm 1 , $a_{k}(t)$ is the spreading sequence of user $k, T_{b}$ is the symbol period, and $\tau_{k}$ is the propagation delay of user $k$.

The spreading sequence of user $k$ is defined as $a_{k}(t)=\sum_{m=1}^{N} a_{k, m} \psi\left(t-m T_{c}\right)$, where $a_{k, m}$ is the spreading code, $T_{c}$ is the chip period, $N$ is the length of the spreading code in terms of chip periods, and $\psi(t)$ is the transmitted pulse shape. Each user's transmitted signal is assumed to pass through a frequency-selective Rayleigh fading channel. Additive white Gaussian noise (AWGN) is also considered with 


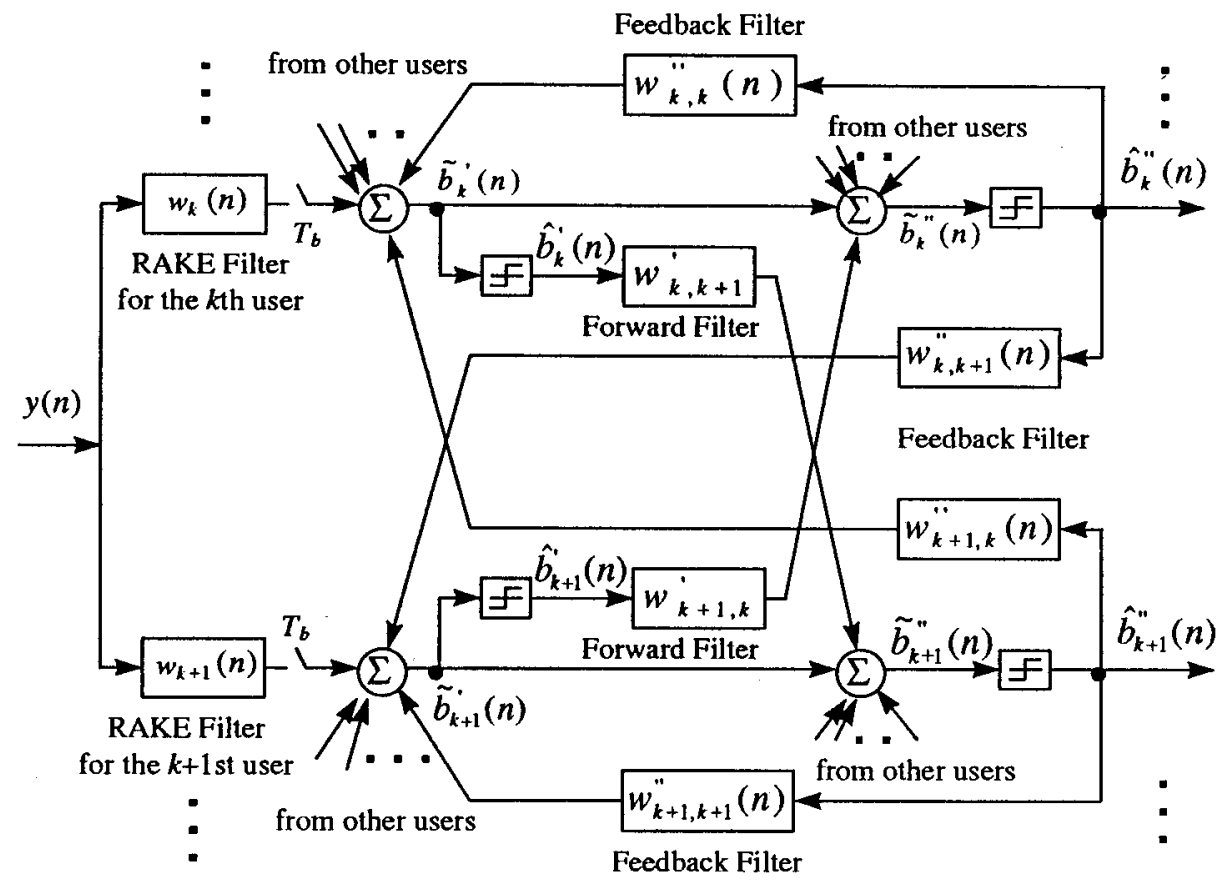

Fig. 2. Structure of the proposed detector.

two-sided power spectral density $N_{0} / 2$. In the base station, the receiver signal can be represented as

$$
y^{c}(t)=\sum_{k=1}^{K} y_{k}^{c}(t)+v^{c}(t)
$$

where

$$
\begin{aligned}
y_{k}^{c}(t) & =\sum_{n} b_{k}(n) \sum_{l=0}^{L_{k}-1} c_{k}(l) a_{k}\left(t-n T_{b}-\tau_{k}-t_{l ; k}\right) \\
& =\sum_{n} b_{k}(n) h_{k}^{c}\left(t-n T_{b}\right)
\end{aligned}
$$

and

$$
h_{k}^{c}(t)=\sum_{l=0}^{L_{k}-1} c_{k}(l) a_{k}\left(t-\tau_{k}-t_{l ; k}\right)
$$

$L_{k}$ is the number of paths of user $k$, the complex quantity $c_{k}(l)$ is the amplitude and phase variation of the $l$ th path of user $k, t_{l ; k}$ is the reception time of the $l$ th path of user $k$, and $h_{k}^{c}(t)$ denotes the overall impulse response of channel including the spreading sequence $a_{k}(t)$. In the base station, all the multiplexed SS signals, except the desired SS signal, interfere with the desired SS signal, owning to cross correlation of different pseudonoise (PN) sequences assigned to individual users. Therefore, in general, it is necessary to use a set of PN sequences with a sufficiently small cross correlation. Nevertheless, as the number of interfering users increases, the amount of MAI increases. In addition, the presence of strong users exacerbates the MAI of the weaker users.

\section{B. Structure of the Adaptive Detector}

This study concentrates on developing a detector design such that the effects of ISI and MAI are reduced, and at the same time, the multipath diversity and processing gain can be exploited with less noise enhancement. We now describe an adaptive detector which can adaptively cancel ISI as well as MAI in an asynchronous CDMA system without a priori channel knowledge, i.e., from the receiver signal $y^{c}(t)$ to estimate all the transmitted symbol $b_{k}(n)(k=1 \cdots K)$ simultaneously. The proposed detector is shown in Fig. 2.

If the received signal is sampled every $T_{f}\left(T_{f}=T_{c} / p\right)$ interval, then the discrete-time received signal is

$$
\begin{aligned}
y(n) & =\left.y^{c}(t)\right|_{t=n T_{f}} \\
& =\sum_{j=1}^{K} \sum_{m} b_{j}(m) h_{j}^{c}\left(n T_{f}-m T_{b}\right)+v^{c}\left(n T_{f}\right) \\
& =\sum_{j=1}^{K} \sum_{m} b_{j}(m) h_{j}(n-m p N)+v(n)
\end{aligned}
$$

where $h_{j}(n)=h_{j}^{c}\left(n T_{f}\right)$ is a sampled version of continuous time impulse response, and $v(n)=v^{c}\left(n T_{f}\right)$ represents a sequence of additive noise samples.

Remark 1: In practical application, the sampling interval $T_{f}=T_{c} / p$, where $p$ is an integer. Due to the fractional space structure of the receiver, the detector does not require knowledge of any user's spreading code or timing information [21].

ISI arises for $L_{k}$ greater than one and MAI arises due to channel distortion or nonorthogonal spreading sequences $a_{k, m}$ or both. Therefore, the receiver sequences $y(n)$ have to be processed in a detector to estimate the transmitted data symbol sequences $b_{k}(n)$. In order to do this, the detector estimates and subtracts out all of the ISI and MAI for each user in parallel, which consists of RAKE filters, forward filters, and feedback filters. The RAKE filter acts as a multipath diversity combiner as well as a canceller of precursor ISI and precursor MAI. The 
forward filter acts as a canceller of main-cursor MAI. The feedback filter acts as a canceller of postcursor ISI and postcursor MAI.

In the $k$ th user, the sampled signal $y(n)$ is fed to the RAKE filter. The RAKE filter output $z_{k}(n)$ at the $n$th symbol interval, which is calculated every $T_{b}$ interval, is given by

$$
\begin{aligned}
z_{k}(n)= & Y(n) W_{k} \\
= & \sum_{i} y(n p N-i) w_{k}(i) \\
= & \sum_{j=1}^{K} \sum_{m} b_{j}(m) \sum_{i} h_{j}(n p N-i-m p N) w_{k}(i) \\
& +\sum_{i} v(n p N-i) w_{k}(i) \\
= & b_{k}(n) \sum_{i} h_{j}(-i) w_{k}(i) \\
& +\sum_{i=1, j \neq k}^{K} b_{j}(n) \sum_{i} h_{j}(-i) w_{k}(i) \\
& +\sum_{j=1}^{K} \sum_{m<n} b_{j}(m) \sum_{i} h_{j}(n p N-i-m p N) w_{k}(i) \\
& +\sum_{j=1}^{K} \sum_{m>n} b_{j}(m) \sum_{i} h_{j}(n p N-i-m p N) w_{k}(i) \\
& +\sum_{i} v(n p N-i) w_{k}(i)
\end{aligned}
$$

where $W_{k}$ and $Y(n)$, which are vectors of the tap-coefficients of RAKE filter for the $k$ th user and receiver signal, respectively, are given by

$$
\begin{aligned}
Y(n) & =\left[y\left(n p N+L_{1}\right), \cdots, y(n p N), \cdots, y\left(n p N-L_{2}\right)\right] \\
W_{k} & =\left[w_{k}\left(-L_{1}\right), \cdots, w_{k}(0), \cdots, w_{k}\left(L_{2}\right)\right]^{T}
\end{aligned}
$$

where the superscript $T$ denotes transposition.

In (12), the desired symbol is in first term, the second term is main-cursor MAI, the third term contains postcursor ISI $(j=$ $k$ ) and postcursor MAI $(j \neq k)$, and the fourth term contains precursor ISI $(j=k)$ and precursor MAI $(j \neq k)$.

The initial symbol estimate, denoted by $\tilde{b}_{k}^{\prime}(n)$, is obtained by substracting both the previously estimated postcursor MAI symbols $\left\{\hat{b}_{j}^{\prime \prime}(\cdot)\right\}$ (for $j=1 \cdots K, j \neq k$ ) and the estimated postcursor ISI symbol $\left\{\hat{b}_{k}^{\prime \prime}(\cdot)\right\}$ from the RAKE filter output $z_{k}(n)$, that is

$$
\tilde{b}_{k}^{\prime}(n)=z_{k}(n)+\hat{B}_{t}^{\prime \prime}(n-1) W_{k}^{\prime \prime}
$$

where $W_{k}^{\prime \prime}$ and $\hat{B}_{t}^{\prime \prime}(n)$, which are vectors of tap-coefficients of the feedback filter for the $k$ th user and a previous symbol estimation, respectively, given by

$$
\begin{aligned}
\hat{B}_{t}^{\prime \prime}(n)= & {\left[\hat{b}_{1}^{\prime \prime}(n), \cdots, \hat{b}_{1}^{\prime \prime}\left(n-L_{3}+1\right), \cdots,\right.} \\
& \left.\hat{b}_{K}^{\prime \prime}(n), \cdots, \hat{b}_{K}^{\prime \prime}\left(n-L_{3}+1\right)\right] \\
W_{k}^{\prime \prime}= & {\left[w_{1 ; k}^{\prime \prime}(1), \cdots, w_{1 ; k}^{\prime \prime}\left(L_{3}\right), \cdots,\right.} \\
& \left.w_{K ; k}^{\prime \prime}(1), \cdots, w_{K ; k}^{\prime \prime}\left(L_{3}\right)\right]^{T}
\end{aligned}
$$

The feedback filter $w_{j, k}^{\prime \prime}(\cdot)$ represent the tap-coefficients which multiply the respective detected symbols coming from the $j$ th user. An initial decision output denoted by $\hat{b}_{k}^{\prime}(n)$, is obtained by the first decision device, i.e.,

$$
\hat{b}_{k}^{\prime}(n)=\operatorname{sgn}\left(\operatorname{Re}\left\{\tilde{b}_{k}^{\prime}(n)\right\}\right)
$$

where sgn and Re denote the sign function and real part, respectively.

The feedback filter only cancels both the postcursors of ISI and postcursor MAI from the output of RAKE filter. Therefore, if the RAKE filter can exploit the multipath diversity and processing gain, as well as suppress both precursor MAI and precursor ISI, the output signal $\tilde{b}_{k}^{\prime}(n)$ will only contain the desired symbol and main-cursor MAI.

Next, we use the initial decisions, $\hat{b}_{j}^{\prime}(n)$ (for $j=1 \cdots K$ ), to cancel the main-cursor MAI. That is, the signal $\hat{b}_{j}^{\prime}(n)(j \neq k)$ is fed to the forward filter $w_{j ; k}^{\prime}$ to cancel the main-cursor MAI and then a final decision $\hat{b}_{k}^{\prime \prime}(n)$ is obtained by the final decision device, i.e.,

$$
\begin{aligned}
& \tilde{b}_{k}^{\prime \prime}(n)=\tilde{b}_{k}^{\prime}(n)+\hat{B}_{k}^{\prime}(n) W_{k}^{\prime} \\
& \hat{b}_{k}^{\prime \prime}(n)=\operatorname{sgn}\left(\operatorname{Re}\left\{\tilde{b}_{k}^{\prime \prime}(n)\right\}\right)
\end{aligned}
$$

where $W_{k}^{\prime}$ and $\hat{B}_{k}^{\prime}(n)$ are the tap-coefficients vectors of the forward filter for the $k$ th user and an initial symbol estimation, respectively, and are given by

$$
\begin{gathered}
\hat{B}_{k}^{\prime}(n)=\left[\hat{b}_{1}^{\prime}(n), \cdots, \hat{b}_{k-1}^{\prime}(n), \hat{b}_{k+1}^{\prime}(n), \cdots, \hat{b}_{K}^{\prime}(n)\right] \\
W_{k}^{\prime}=\left[w_{1, k}^{\prime}, \cdots, w_{k-1, k}^{\prime}, w_{k+1, k}^{\prime}, \cdots, w_{K, k}^{\prime}\right]^{T} .
\end{gathered}
$$

Remark 2: The RAKE filter not only exploits multipath diversity and processing gain, but also supresses the precursor MAI and precursor ISI. Thus, the RAKE filter is similar to a RAKE combiner-canceller [20].

We proposed both of the decisions $\left\{\hat{b}^{\prime}(\cdot)\right\}$ and $\left\{\hat{b}^{\prime \prime}(\cdot)\right\}$ to cancel the MAI and ISI by using forward filters and feedback filters, respectively. Since the forward filters and feedback filters work in noise-free environments [18], thus both filters cancel MAI and ISI without noise enhancement, thereby imparting the RAKE filters with great flexibility in handling multipath diversity and processing gain. We can take advantage of this factor to design the detector based on MMSE criterion. More details of this design are given in the following section.

\section{MMSE ESTIMATION}

\section{A. Criterion}

A potential problem of performance deterioration with the proposed detector occurs if the initial data estimates are not reliable. In this case, the subtraction of an interfering bit based on an incorrect bit estimate causes a quadrupling in the interfering power for that bit. Thus, too many incorrect initial data estimates may cause severe performance degradation. Therefore, a cost function based on error signals $\mathcal{E}_{1}(n)$ and $\mathcal{E}_{2}(n)$ is chosen 
to specify the tap-coefficients of all filters to improve the performance of the detector. In this case, the error signals $\mathcal{E}_{1}(n)$ and $\mathcal{E}_{2}(n)$ are defined as the difference between the estimated symbols and desired symbols $b_{j}(n)(j=1 \cdots K)$; that is

$$
\mathcal{E}_{1}(n)=B^{T}(n)-\tilde{B}^{\prime T}(n)
$$

and

$$
\mathcal{E}_{2}(n)=B^{T}(n)-\tilde{B}^{\prime \prime T}(n)
$$

where $B(n)=\left[b_{1}(n), \cdots, b_{K}(n)\right], \tilde{B}^{\prime}(n)=\left[\tilde{b}_{1}^{\prime}(n), \cdots\right.$, $\left.\tilde{b}_{K}^{\prime}(n)\right]$ and $\tilde{B}^{\prime \prime}(n)=\left[\tilde{b}_{1}^{\prime \prime}(n), \cdots, \tilde{b}_{K}^{\prime \prime}(n)\right]$.

The cost function can be defined as

$$
\begin{aligned}
J(n)= & \gamma E\left\{\left|\mathcal{E}_{1}(n)\right|^{2}\right\}+E\left\{\left|\mathcal{E}_{2}(n)\right|^{2}\right\} \\
= & \gamma E\left\{\sum_{j=1}^{K}\left|b_{j}(n)-Y(n) W_{j}-\hat{B}_{t}^{\prime \prime}(n-1) W_{j}^{\prime \prime}\right|^{2}\right\} \\
+ & E\left\{\sum_{j=1}^{K} \mid b_{j}(n)-Y(n) W_{j}-\hat{B}_{t}^{\prime \prime}(n-1) W_{j}^{\prime \prime}\right. \\
& \left.-\left.\hat{B}_{j}^{\prime}(n) W_{j}^{\prime}\right|^{2}\right\}
\end{aligned}
$$

where $\gamma$ is a weighting factor and $\gamma \geq 0$.

The tap-coefficients of all filters are chosen to minimize the cost function. The optimal solution can be obtained by solving the following equations (under the assumption of perfect decision):

$$
\frac{\partial J(n)}{\partial W_{k}}=O, \quad \frac{\partial J(n)}{\partial W_{k}^{\prime}}=O, \quad \text { and } \quad \frac{\partial J(n)}{\partial W_{k}^{\prime \prime}}=O .
$$

Therefore the optimum tap-coefficient estimates of all filters are expressed by

$$
\begin{aligned}
W_{k_{\mathrm{opt}}} & =\left[R_{Y Y}-\frac{R_{B_{k}^{\prime} Y}^{H} R_{B_{k}^{\prime} Y}}{1+\gamma}-R_{Y B_{t}^{\prime \prime}} R_{Y B_{t}^{\prime \prime}}^{H}\right]^{-1} \cdot R_{b_{k} Y}^{H} \\
W_{k_{\mathrm{opt}}}^{\prime} & =-R_{B_{k}^{\prime} Y} W_{k_{\mathrm{opt}}} \\
W_{k_{\mathrm{opt}}}^{\prime \prime} & =-R_{Y B_{t}^{\prime \prime}}^{H} W_{k_{\mathrm{opt}}}
\end{aligned}
$$

where superscript $H$ denotes Hermitian transposition, and

$$
\begin{aligned}
R_{b_{k} Y} & =E\left[b_{k}^{H}(n) Y(n)\right] \\
R_{b_{k} B_{t}^{\prime \prime}} & =E\left[b_{k}^{H}(n) B_{t}^{\prime \prime}(n-1)\right] \\
& =O \\
R_{Y Y} & =E\left[Y^{H}(n) Y(n)\right] \\
R_{Y B_{t}^{\prime \prime}} & =E\left[Y^{H}(n) B_{t}^{\prime \prime}(n-1)\right] \\
R_{B_{k}^{\prime} b_{k}} & =E\left[B_{k}^{\prime H}(n) b_{k}(n)\right] \\
& =O \\
R_{B_{k}^{\prime} Y} & =E\left[B_{k}^{\prime H}(n) Y(n)\right]
\end{aligned}
$$

$$
\begin{aligned}
R_{B_{k}^{\prime} B_{t}^{\prime \prime}} & =E\left[B_{k}^{\prime H}(n) B_{t}^{\prime \prime}(n-1)\right] \\
& =O \\
R_{B_{k}^{\prime} B_{k}^{\prime}} & =E\left[B_{k}^{\prime H}(n) B_{k}^{\prime}(n)\right] \\
& =I \\
R_{B_{t}^{\prime \prime} B_{t}^{\prime \prime}} & =E\left[B_{t}^{\prime \prime H}(n-1) B_{t}^{\prime \prime}(n-1)\right] \\
& =I .
\end{aligned}
$$

Substituting $W_{k_{\mathrm{opt}}}, W_{k_{\mathrm{opt}}}^{\prime}$, and $W_{k_{\mathrm{opt}}}^{\prime \prime}$ into (26), the MMSE solution, $\xi_{1_{\mathrm{opt}}}$ and $\xi_{2_{\mathrm{opt}}}$ of $\mathcal{E}_{1}(n)$ and $\mathcal{E}_{2}(n)$, respectively, are found to be equal to

$$
\begin{aligned}
& \xi_{1_{\mathrm{opt}}}=\sum_{j=1}^{K}\left[1-R_{b_{k} Y} W_{j_{\mathrm{opt}}}+\frac{1}{1+\gamma}\right. \\
& \left.\cdot W_{j_{\mathrm{opt}}}^{H} R_{B_{j}^{\prime} Y}^{H} R_{B_{j}^{\prime} Y} W_{j_{\mathrm{opt}}}\right] \\
& \xi_{2_{\mathrm{opt}}}=\sum_{j=1}^{K}\left[1-R_{b_{k} Y} W_{j_{\mathrm{opt}}}-\frac{\gamma}{1+\gamma}\right. \\
& \left.\cdot W_{j_{\mathrm{opt}}}^{H} R_{B_{j}^{\prime} Y}^{H} R_{B_{j}^{\prime} Y} W_{j_{\mathrm{opt}}}\right]
\end{aligned}
$$

and the optimal $J(n)$ in (26) is obtained as $J_{\mathrm{opt}}=\gamma \xi_{1_{\mathrm{opt}}}+\xi_{2_{\mathrm{opt}}}$.

From (28), if $\gamma=0$, the $W_{k_{\text {opt }}}$ only suppresses the percursor MAI and precursor ISI as well as exploits multipath diversity and processing gain, i.e., $\tilde{b}^{\prime}(n)$ remains the total maincursor MAI. This may cause erroneous initial decisions. For this reason, we can propose $\gamma>0$ in (26), so that $W_{k_{\text {opt }}}$ can contribute the main-cursor MAI cancellation. For the larger values of $\gamma$, the larger part of main-cursor MAI could be cancelled by $W_{j_{\text {opt }}}$. In this situation, it would lead to a more reliable initial decision. But as $\gamma$ increases, the loading of the forward filter $W_{k_{\text {opt }}}$ will increase, thus losing the ability of multipath diversity (including processing gain) and causing noise enhancement, i.e., it will degrade SNR.

As mentioned above, owing to the tradeoff between erroneous initial decision and SNR protection, the selection of $\gamma$ is very important factor in the design of the proposed detector.

\section{B. Estimation Using Recursive Least Squares (RLS) Algorithm}

Since the matrices $R_{Y Y}, R_{B_{k}^{\prime} Y}, R_{Y B_{t}^{\prime \prime}}$, and $R_{b_{k} Y}$ cannot be obtained easily from the received signal, and in addition, in the CDMA system the channel impulse response $c_{k}(l)$ may be time varying. Therefore, the minimization of the MSE of the error signal can be performed by searching the tap-coefficients using an RLS algorithm. From (15) and (19), both output $\tilde{B}^{\prime}(n)$ and $\tilde{B}^{\prime \prime}(n)$ can be represented as shown in (42) at the bottom of the next page, or

$$
\tilde{\mathcal{B}}(n)=:\left[\frac{\mathcal{H}_{1}(n)}{\mathcal{H}_{2}(n)}\right] \mathcal{X}=\mathcal{H}(n) \mathcal{X}
$$

where $\mathcal{H}(n)$ and $\mathcal{X}$ are input matrix and state vector, respectively.

The RLS algorithm is proposed to update the tap-coefficients $\hat{\mathcal{X}}(n)$ and estimate the vectors of symbol $\tilde{B}^{\prime}(n)$ and $\tilde{B}^{\prime \prime}(n)$ for 
all users. The following equation presents a summary of RLS algorithms (see Appendix):

$$
\begin{aligned}
& \tilde{\mathcal{B}}(n)=\mathcal{H}(n) \hat{\mathcal{X}}(n-1) \\
& \mathcal{K}(n)=\mathcal{P}(n-1) \mathcal{H}^{H}(n)\left[\mathcal{H}(n) \mathcal{P}(n-1) \mathcal{H}^{H}(n)+\lambda \mathcal{C}\right]^{-1} \\
& \hat{\mathcal{X}}(n)=\hat{\mathcal{X}}(n-1)+\mathcal{K}(n)\left[\hat{\mathcal{B}}(n)-\tilde{\mathcal{B}}^{\prime}(n)\right] \\
& \quad \text { for tracking period } \\
& \hat{\mathcal{X}}(n)=\hat{\mathcal{X}}(n-1)+\mathcal{K}(n)\left[\mathcal{B}(n)-\tilde{\mathcal{B}}^{\prime}(n)\right] \\
& \quad \text { for training period } \\
& \mathcal{P}(n)=\lambda^{-1}[I-\mathcal{K}(n) \mathcal{H}(n)] \mathcal{P}(n-1)
\end{aligned}
$$

where $\lambda$ is the forgetting factor, with $0<\lambda \leq 1$, and $\mathcal{C}$ has the following form:

$$
\mathcal{C}=\left[\begin{array}{cc}
I / \gamma & O \\
O & I
\end{array}\right] .
$$

After the tap-coefficients of RAKE filter, forward filter and feedback filter of the proposed detector are estimated by employing the above RLS algorithm, the estimation of transmitted sequences by the proposed detector is given by

$$
\tilde{\mathcal{B}}(n)=\mathcal{H}(n) \hat{\mathcal{X}}(n-1)
$$

and

$$
\hat{\mathcal{B}}(n)=\operatorname{sgn}(\operatorname{Re}\{\tilde{\mathcal{B}}(n)\}) .
$$

\section{The Choice of Weighting Factor}

In the detection algorithm as analyzed in above section, the selection of weighting factor $\gamma$ in (26) is very important for the BER performance of the proposed detector. In practice, we cannot get a priori knowledge of $\gamma$. In addition, the transmission channel may be time varying, hence, it is impossible for $\gamma$ to be a constant in the proposed detector. From extensive simulations, we have found that if the $\xi_{1}$ does not exceed $\xi_{2}$ by $2.5 \mathrm{~dB}$, the initial decision $\hat{b}_{k}^{\prime}(n)$ is reliable. Thus, in order to clarify how to select an adequate $\gamma$, a design procedure can be outlined as follows.

Step1: Choose a large $\gamma$ to let $\xi_{1}$ approach $\xi_{2}$, with less erroneous initial symbol estimation as in the previous analysis.
Step2: Decrease the value of $\gamma$ so that $\xi_{1}$ is not larger than $\xi_{2}$ by $2.5 \mathrm{~dB}$.

Step3: Increase the value of $\gamma$ if $\xi_{1}$ exceeds $\xi_{2}$ by $2.5 \mathrm{~dB}$ (a large error initial decision is made). Otherwise, decrease the value of $\gamma$.

Here, $\xi_{1}$ and $\xi_{2}$ can be adjusted by the following recursive algorithms, respectively

$$
\xi_{1}(n)=\beta \xi_{1}(n-1)+(1-\beta)\left|\mathcal{E}_{1}(n)\right|^{2}
$$

and

$$
\xi_{2}(n)=\beta \xi_{2}(n-1)+(1-\beta)\left|\mathcal{E}_{2}(n)\right|^{2}
$$

where $0<\beta<1$ determines the memory length of the recursive algorithm.

\section{Convergence Analysis}

It is well known that, for $\lambda=1$ and $n \rightarrow \infty$, the RLS algorithm realizes the optimum Wiener solution $\mathcal{X}_{\text {opt }}$. However, this situation changes drastically if one uses an exponentially windowed RLS algorithm $(\lambda<1)$ in order to track possible variations in the unknown channel. In that case, the RLS algorithm causes noise in the tap-coefficients and causes them to be on the average misadjusted from their optimal values. In the following, we provide a convergence analysis for this algorithm.

Using adaptation (47) along with (A.18) and (A.12), we can easily show that

$$
\Phi(n) \hat{\mathcal{X}}(n)=\lambda \Phi(n-1) \hat{\mathcal{X}}(n-1)+\mathcal{H}^{H}(n)\left[\begin{array}{cc}
\gamma I & O \\
O & I
\end{array}\right] \mathcal{B}(n) .
$$

Denote the optimum error vector and tap-coefficient error at time $n$ as

$$
\begin{aligned}
& \mathcal{E}_{\mathrm{opt}}(n)=: \mathcal{B}(n)-\mathcal{H}(n) \mathcal{X}_{\mathrm{opt}} \\
& \triangle \mathcal{X}(n)=: \hat{\mathcal{X}}(n)-\mathcal{X}_{\mathrm{opt}}
\end{aligned}
$$

By using (55), (56), and (A.12), (54) yields the following recursive relation for the tap-coefficient error vector:

$$
\begin{aligned}
& \triangle \mathcal{X}(n)=\lambda \Phi^{-1}(n) \Phi(n-1) \triangle \mathcal{X}(n-1) \\
& +\Phi^{-1}(n) \mathcal{H}^{H}(n)\left[\begin{array}{cc}
\gamma I & O \\
O & I
\end{array}\right] \mathcal{E}_{\mathrm{opt}}(n)
\end{aligned}
$$


Taking expected values on both sides of above equation, we have

$$
\begin{aligned}
E\{\triangle \mathcal{X}(n)\} & =\lambda E\left\{\Phi^{-1}(n) \Phi(n-1) \triangle \mathcal{X}(n-1)\right\} \\
& \approx \lambda E\{\triangle \mathcal{X}(n-1)\} \quad \text { as } n \rightarrow \infty
\end{aligned}
$$

The fact that the optimum error vectors $\mathcal{E}_{\text {opt }}(n)$ and $\mathcal{E}_{2_{\text {opt }}}(n)$ are orthogonal with $\mathcal{H}_{1}(n)$ and $\mathcal{H}_{2}(n)$, respectively, and $\Phi^{-1}(n) \approx(1-\lambda) \Phi^{-1}$ as $n \rightarrow \infty$ [17] concludes that the expected value of the second term in (57) is approximately zero. Besides, in the steady state, $\Phi(n)$ and $\Phi(n-1)$ are almost equal [17], i.e., $\Phi^{-1}(n) \Phi(n-1) \approx I$ as $n \rightarrow \infty$.

By the fact $0<\lambda<1$, we conclude $E\{\triangle \mathcal{X}(n)\} \rightarrow 0$ as $n \rightarrow \infty$. Therefore, the expected values for the tap-coefficient error vector always converge to zero, and this convergence is independent of the eigenvalue distribution of covariance matrix of input signal.

Next, we consider the convergence of the MSE.

$$
\begin{aligned}
\xi_{1}(n)= & E\left\{\left|B^{T}(n)-\mathcal{H}_{1}(n) \mathcal{X}(n)\right|^{2}\right\} \\
= & E\left\{\mid B^{T}(n)-\mathcal{H}_{1}(n) \mathcal{X}_{\mathrm{opt}}(n)\right. \\
& \left.-\left.\mathcal{H}_{1}(n) \triangle \mathcal{X}(n)\right|^{2}\right\} \\
= & \xi_{1_{\mathrm{opt}}}+\frac{1}{\gamma} E\left\{\triangle \mathcal{X}^{H}(n) \Phi_{1} \triangle \mathcal{X}(n)\right\}
\end{aligned}
$$

Similarly

$$
\xi_{2}=\xi_{2 \mathrm{opt}}+E\left\{\triangle \mathcal{X}^{H}(n) \Phi_{2} \triangle \mathcal{X}(n)\right\}
$$

where

$$
\begin{aligned}
& \Phi_{1}=E\left\{\gamma \mathcal{H}_{1}^{H}(n) \mathcal{H}_{1}(n)\right\} \quad \text { and } \\
& \Phi_{2}=E\left\{\mathcal{H}_{2}^{H}(n) \mathcal{H}_{2}(n)\right\} .
\end{aligned}
$$

Therefore, $\quad(1 / \gamma) E\left\{\triangle \mathcal{X}^{H}(n) \Phi_{1} \quad \triangle \quad \mathcal{X}(n)\right\} \quad$ and $E\left\{\triangle \mathcal{X}^{H}(n) \Phi_{2} \triangle \mathcal{X}(n)\right\}$ are the excess MSE's of $\xi_{1}(n)$ and $\xi_{2}(n)$, respectively.

Now, we are interested in the asymptotic behavior of the average excess MSE's. Substituting for $\triangle \mathcal{X}(n)$ in (57), we obtain

$$
\begin{aligned}
E\{ & \left.\triangle \mathcal{X}^{H}(n) \Phi_{1} \triangle \mathcal{X}(n)\right\} \\
= & \lambda^{2} E\left\{\triangle \mathcal{X}^{H}(n-1) \Phi^{H}(n-1) \Phi^{-1}(n) \Phi_{1} \Phi^{-1}(n)\right. \\
& \cdot \Phi(n-1) \triangle \mathcal{X}(n-1)\} \\
& +\lambda E\left\{\triangle \mathcal{X}^{H}(n-1) \Phi(n-1)\right. \\
& \left.+\Phi^{-1}(n) \Phi_{1} \Phi^{-1}(n) \mathcal{H}^{H}(n)\left[\begin{array}{cc}
\gamma I & O \\
O & I
\end{array}\right] \mathcal{E}_{\mathrm{opt}}(n)\right\} \\
& +\lambda E\left\{\mathcal{E}_{\mathrm{opt}}^{H}(n)\left[\begin{array}{cc}
\gamma I & O \\
O & I
\end{array}\right] \mathcal{H}(n) \Phi^{-1}(n) \Phi_{1} \Phi^{-1}(n)\right. \\
& \left.+\Phi(n-1) \triangle \mathcal{X}^{H}(n-1)\right\} \\
& +E\left\{\mathcal{E}_{\mathrm{opt}}^{H}(n)\left[\begin{array}{cc}
\gamma I & O \\
O & I
\end{array}\right] \mathcal{H}(n)\right. \\
& \left.\cdot \Phi^{-1}(n) \Phi_{1} \Phi^{-1}(n) \mathcal{H}^{H}(n)\left[\begin{array}{cc}
\gamma I & O \\
O & I
\end{array}\right] \mathcal{E}_{\mathrm{opt}}(n)\right\} \cdot(65)
\end{aligned}
$$

In a steady state, $\Phi^{-1}(n) \Phi(n-1) \approx I$ and $\Phi^{-1}(n) \approx(1-$ 1) $\Phi^{-1}[17]$. Furthermore, the optimum error vectors $\mathcal{E}_{1_{\text {opt }}}(n)$ and $\mathcal{E}_{1_{\text {opt }}}(n)$ are orthogonal with $\mathcal{H}_{1}(n)$ and $\mathcal{H}_{2}(n)$, respectively. With the assumption that $\triangle \mathcal{X}(n-1)$ is independent of $\mathcal{H}(n)$ and $\mathcal{E}_{\text {opt }}(n)$, the expected values of the second and third term in the above equation are approximately zero, i.e.,

$$
\begin{aligned}
E\{ & \left.\triangle \mathcal{X}^{H}(n) \Phi_{1} \triangle \mathcal{X}(n)\right\} \\
= & \lambda^{2} E\left\{\triangle \mathcal{X}^{H}(n-1) \Phi_{1} \triangle \mathcal{X}(n-1)\right\} \\
& +E\left\{\mathcal{E}_{\text {opt }}^{H}(n)\left[\begin{array}{cc}
\gamma I & O \\
O & I
\end{array}\right] \mathcal{H}(n) \Phi^{-1}(n)\right. \\
& \left.\times \Phi_{1} \Phi^{-1}(n) \mathcal{H}^{H}(n) \cdot\left[\begin{array}{cc}
\gamma I & O \\
O & I
\end{array}\right] \mathcal{E}_{\mathrm{opt}}(n)\right\} \\
= & \lambda^{2} E\left\{\triangle \mathcal{X}^{H}(n-1) \Phi_{1} \Delta \mathcal{X}(n-1)\right\} \\
& +(1-\lambda)^{2} \operatorname{tr}\left\{E \left\{\Phi^{-1} \mathcal{H}^{H}(n)\left[\begin{array}{cc}
\gamma I & O \\
O & I
\end{array}\right]\right.\right. \\
& \left.\left.\times \mathcal{E}_{\text {opt }}(n) \mathcal{E}_{\text {opt }}^{H}(n) \cdot\left[\begin{array}{cc}
\gamma I & O \\
O & I
\end{array}\right] \mathcal{H}(n) \Phi^{-1}\right\} \Phi_{1}\right\} .
\end{aligned}
$$

Let us denote

$$
\begin{aligned}
\xi_{1}^{\prime}=\operatorname{tr}\left\{E \left\{\Phi^{-1} \mathcal{H}^{H}(n)\right.\right. & {\left[\begin{array}{cc}
\gamma I & O \\
O & I
\end{array}\right] \mathcal{E}_{\mathrm{opt}}(n) \mathcal{E}_{\mathrm{opt}}^{H}(n) } \\
& \left.\left.\cdot\left[\begin{array}{cc}
\gamma I & O \\
O & I
\end{array}\right] \mathcal{H}(n) \Phi^{-1}\right\} \Phi_{1}\right\} .
\end{aligned}
$$

Then, (67) becomes

$$
\begin{aligned}
E\{ & \left.\triangle \mathcal{X}^{H}(n) \Phi_{1} \triangle \mathcal{X}(n)\right\} \\
& =\lambda^{2} E\left\{\triangle \mathcal{X}^{H}(n-1) \Phi_{1} \triangle \mathcal{X}(n-1)\right\}+(1-\lambda)^{2} \xi_{1}^{\prime} .
\end{aligned}
$$

Since $\lambda^{2}<1$, the above equation $E\left\{\triangle \mathcal{X}^{H}(n) \Phi_{1} \triangle \mathcal{X}(n)\right\}$ converges to $\left((1-\lambda) \xi_{1}^{\prime}\right) /(1+\lambda)$ as $n$ tends to $\infty$.

Similarly, let us denote

$$
\begin{aligned}
& \xi_{2}^{\prime}=\operatorname{tr}\left\{E \left\{\Phi^{-1} \mathcal{H}^{H}(n)\right.\right. {\left[\begin{array}{cc}
\gamma I & O \\
O & I
\end{array}\right] \mathcal{E}_{\mathrm{opt}}(n) \mathcal{E}_{\mathrm{opt}}^{H}(n) } \\
&\left.\left.\cdot\left[\begin{array}{cc}
\gamma I & O \\
O & I
\end{array}\right] \mathcal{H}(n) \Phi^{-1}\right\} \Phi_{2}\right\} .
\end{aligned}
$$

Then, we get $E\left\{\triangle \mathcal{X}^{H}(n) \Phi_{2} \triangle \mathcal{X}(n)\right\}$ converges to $\left((1-\lambda) \xi_{2}^{\prime}\right) /(1+\lambda)$ as $n$ tends to $\infty$.

Therefore, we conclude that both average excess MSE's converge.

\section{Simulation Results}

In this section, several simulation examples are presented to illustrate the performance of the proposed adaptive detector in DS-CDMA systems. The following assumptions are made regarding the data symbols, the signature sequences, the channel-impulse response, and noise in simulation.

1) Data symbols: the transmitted data symbol sequences $\left\{b_{j}(n)\right\}, j=1 \cdots K$ are assumed to be uncorrelated BPSK data symbols.

2) Signature sequences: the modified Walsh codes are used as the spreading sequences shown in Table I. We do not claim that modified Walsh codes are the optimum spreading codes for our system since we are dealing with 
TABLE I

MODIFIED WALSH CODE

\begin{tabular}{rrrrrrrr}
\hline 1 & 1 & -1 & -1 & -1 & -1 & -1 & -1 \\
\hline-1 & -1 & 1 & 1 & -1 & -1 & -1 & -1 \\
\hline-1 & -1 & -1 & -1 & 1 & 1 & -1 & -1 \\
\hline 1 & 1 & 1 & 1 & 1 & 1 & -1 & -1 \\
\hline-1 & 1 & 1 & -1 & 1 & -1 & 1 & -1 \\
\hline 1 & -1 & -1 & 1 & 1 & -1 & 1 & -1 \\
\hline 1 & -1 & 1 & -1 & -1 & 1 & 1 & -1 \\
\hline-1 & 1 & -1 & 1 & -1 & 1 & 1 & -1 \\
\hline
\end{tabular}

multipath channels. In this simulation, the number of chips per data symbol interval $(\mathrm{N})$ is equal to 8 .

3) Channel impulse response:

a) the channels all contain three different paths, and all paths are assumed to be equally strong;

b) the uplink is considered, i.e., we assume the location of the paths and delays of the various users are determined randomly within $\left[\begin{array}{ll}0 & 6 T_{c}\end{array}\right]$, $\left[(1 / 4) T_{c} 2 T_{c}\right]$, respectively;

c) the channel coefficients do not change during the data transmission period.

4) Noise: the additive noise is assumed to be white and Gaussian distributed with zero mean; in the following simulation, we fix $E_{b} / N_{0}=6 \mathrm{~dB}$.

The number of RAKE filter taps, feedback filter taps, and forward filter taps are fixed at 30,2, and 1, respectively. A training sequence of 300 data bits is used. It is found that most channels can perform well with a very short training sequence, but we decided to fix the length of the training sequence at 300 bits to simplify the simulation of the proposed method.

In the CDMA system, the goal is for all users to have as low of a BER performance as possible. For illustrating the BER performance of the proposed design algorithm, the average value of BER is defined as

$$
\mathrm{BER}_{\mathrm{av}}=\frac{\sum_{j=1}^{K} \mathrm{BER}_{j}}{K}
$$

where $\mathrm{BER}_{j}$ is the $j$ th user's BER.

\section{A. MMSE and BER Simulation Results}

The MMSE criterion achieves a tradeoff between the error of initial decision and SNR protection by selecting $\gamma$ to minimizing both the output error $\mathcal{E}_{1}(n)$ and $\mathcal{E}_{2}(n)$. In the first example, an asynchronous CDMA system with a number of eight active users is used for the simulation, with all active users having the same power. Fig. 3 shows the MSE curves of $\xi_{1}$ and $\xi_{2}$ with respect to $\gamma$ for the proposed detector. As expected, with a smaller $\gamma$, MSE performance of $\mathcal{E}_{2}(n)$ is better, but $\mathcal{E}_{1}(n)$ has worse MSE performance. A smaller $\gamma$ lets $\tilde{B}^{\prime}(n)$ contain a large amount of main-cursor MAI but allows the RAKE filter $W_{j}$ to preserve the multipath diversity and processing gain to improve SNR of $\tilde{B}^{\prime \prime}(n)$ with less noise enhancement. If we increase the value of $\gamma$, the RAKE filter $W_{j}$ has an additional load to cancel the main-cursor MAI. Therefore, the MSE $\xi_{1}$ of $\mathcal{E}_{1}(n)$ approaches the $\operatorname{MSE} \xi_{2}$ of $\mathcal{E}_{2}(n)$ as $\gamma$ becomes larger. In this sit-

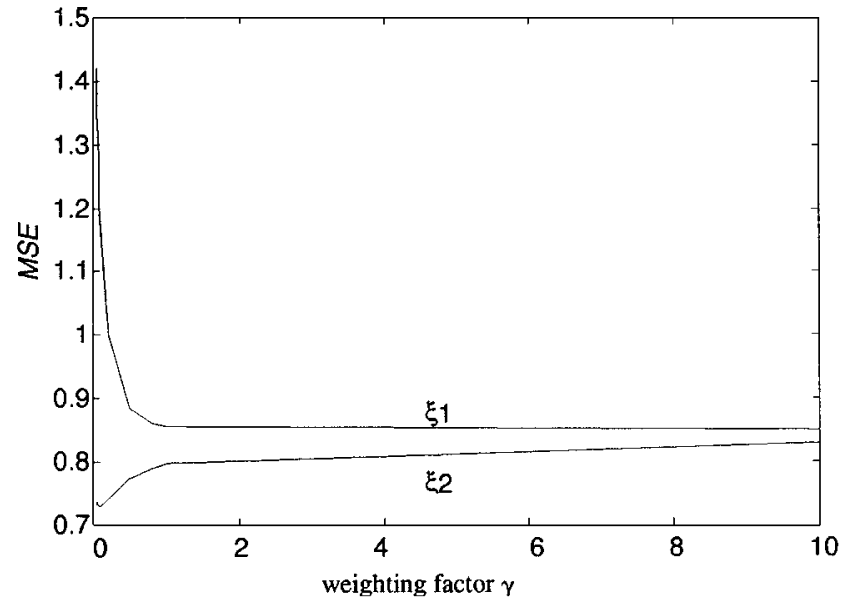

Fig. 3. MSE performance for eight active users.

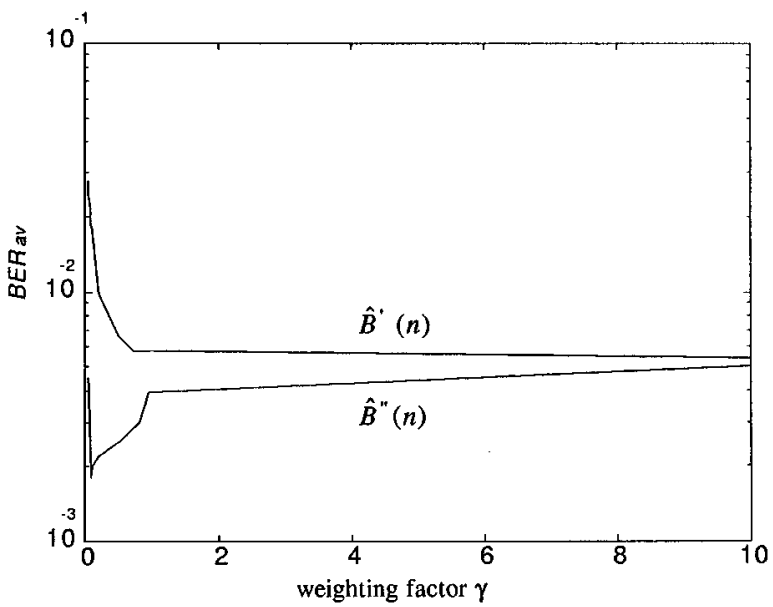

Fig. 4. BER performance for eight active users.

uation, $\tilde{B}^{\prime}(n)$ may contain less main-cursor MAI, but the RAKE filter loses the ability to combine multipath diversity and processing gain to make SNR of $\tilde{B}^{\prime \prime}(n)$ worse and may cause a larger noise enhancement.

Fig. 4 shows the BER performance for the case of eight active users. We can find that the BER of an initial decision $\hat{B}^{\prime}(n)$ is inversely proportional to $\gamma$. However, the BER performance of the final decision $\hat{B}^{\prime \prime}(n)$ has the best performance when $\gamma$ approaches 0.075 , i.e., if $\xi_{2}$ is less than $\xi_{1}$ by $2.5 \mathrm{~dB}$ as shown in Fig. 5 (or $\gamma$ is larger than 0.075) the initial decision becomes reliable in our proposed detector and can achieve a better BER performance in the final decision.

\section{B. Capacity Improvement}

We compare the capacity performance of the proposed canceller with the conventional matched filter (MF) detector and the MMSE-DFE (decision-feedback equalizer) detector [18]. The conventional MF detector consists of a bank of matched filters matched to the spreading codes (assuming the propagation delay is known) and a three-tap RAKE filter, which coherently weights the desired signal multipath components. Fig. 6 shows the BER versus the number $K$ of active users. Figs. 7 and 


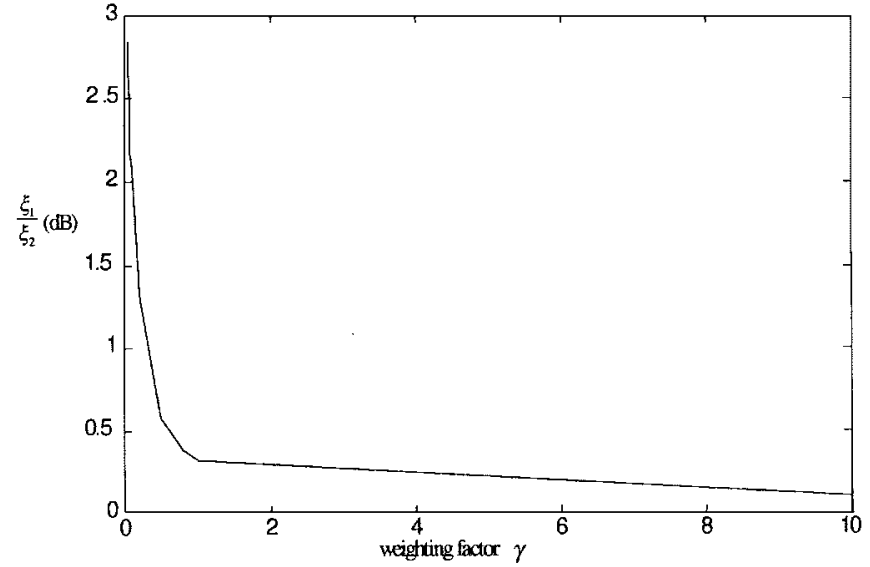

Fig. 5. $\xi_{1} / \xi_{2}$ for eight active users.

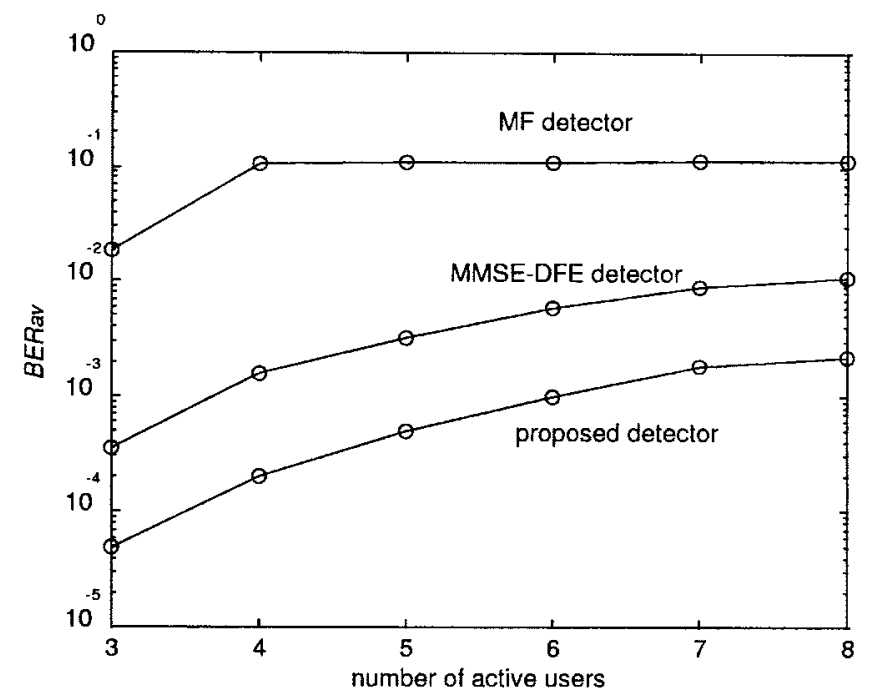

Fig. 6. BER performance versus the number of active users.

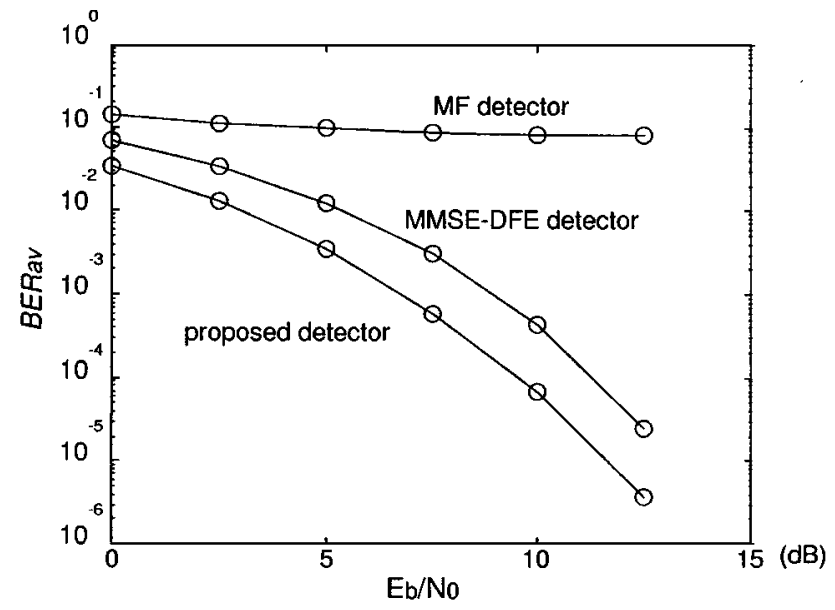

Fig. 7. BER performance versus $E_{b} / N_{0}$.

8 show the BER versus $E_{b} / N_{0}$ for eight active users in multipath channel and ideal (AWGN) channel, respectively. It is clear from these figures that the conventional MF detector and the MMSE-DFE detector provide poor performance in the presence of large number of active users. However, the proposed detector

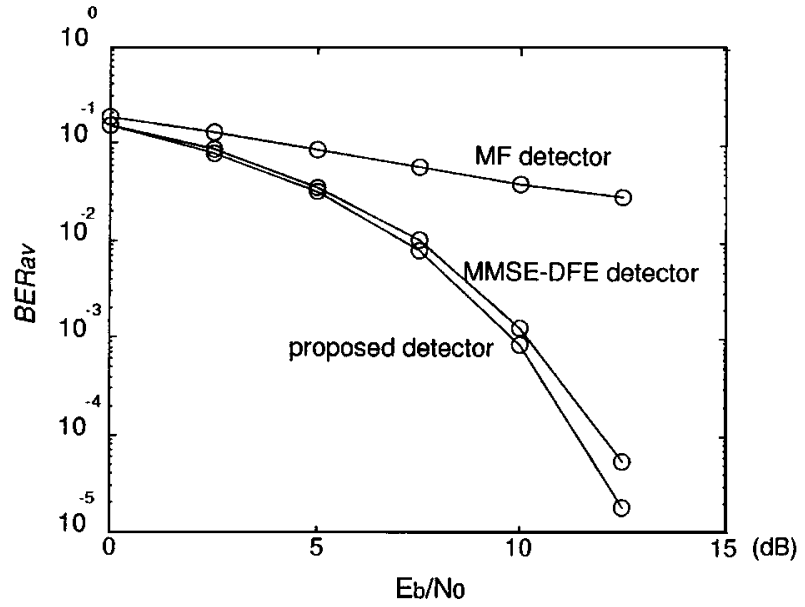

Fig. 8. BER performance versus $E_{b} / N_{0}$.

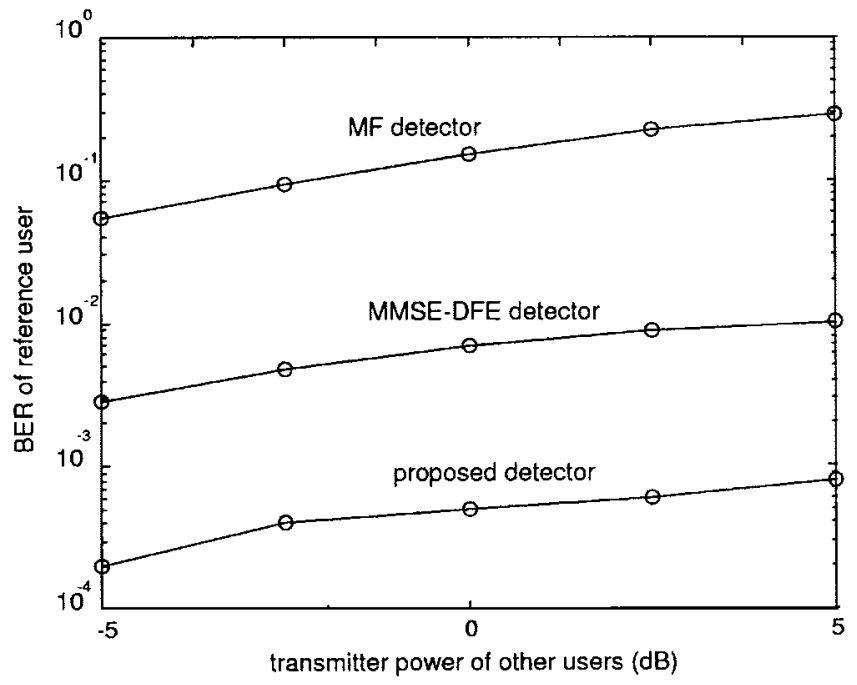

Fig. 9. BER performance versus near-far effect.

can achieve an acceptable BER performance in spite of the increasing number of active users. Since the proposed detector can efficiently cancel the MAI and ISI in multipath fading channel, the transmission capability of the CDMA will be considerably increased.

\section{Near-Far Effect}

The last issue addressed is the sensitivity of the proposed detector to the near-far effect. For this, the transmitter power of reference user (user 1) is kept constant, while the transmitter power of the other users changes from -5 to $+5 \mathrm{~dB}$ with respect to the transmitter power of the reference user. In Fig. 9, it is evident from the BER of reference user results that the proposed detector has good BER performance, whereas the conventional MF detector and MMSE-DFE detector are severely affected by MAI.

\section{CONCLUSION}

In this study, in order to improve the performance of interference suppression for multiuser CDMA transmission systems in multipath fading channels, a new adaptive detector consisting of 
a RAKE filter, forward filter, and feedback filter is introduced to mitigate the effect of ISI and MAI for each user by parallel cancellation. At the same time, the proposed detector preserves multipath diversity and processing gain properties, with less noise enhancement.

An RLS algorithm has been employed to update the tap-coefficients of RAKE filter, forward filter, and feedback filter of the proposed adaptive detector simultaneously. The performance of the proposed parallel cancelling scheme depends heavily on the initial data estimates. Thus, poor initial data estimates may deteriorate the detection performance. A procedure for specifying an adequate weighting factor is also introduced for the RLS algorithm to improve the performance of the proposed adaptive detector.

It has been shown that the adaptive detector has the ability to remove the effect of multipath propagation and MAI, which achieves a significant improvement over conventional MF receivers and MMSE-DFE detectors as the number of active user increases. Consequently, with the use of the proposed adaptive detector, a larger number of active users can simultaneously share a single-channel bandwidth of CDMA systems.

\section{APPENDIX}

DERIVATION OF (44)-(49)

We express the cost function as

$$
\begin{aligned}
J(n)= & \gamma_{1} \sum_{i=1}^{n} \lambda^{n-i}\left|\mathcal{E}_{1}(i)\right|^{2}+\gamma_{2} \sum_{i=1}^{n} \lambda^{n-i}\left|\mathcal{E}_{2}(i)\right|^{2} \\
= & \gamma_{1} \sum_{i=1}^{n} \lambda^{n-i}\left|B^{T}(i)-\mathcal{H}_{1}(i) \mathcal{X}(n)\right|^{2} \\
& +\gamma_{2} \sum_{i=1}^{n} \lambda^{n-i}\left|B^{T}(i)-\mathcal{H}_{2}(i) \mathcal{X}(n)\right|^{2}
\end{aligned}
$$

where $n$ is the variable length of the observed data. In our proposed detector, $\gamma_{1}=\gamma$ and $\gamma_{2}=1$, the vector of error signal is defined as

$$
\begin{aligned}
\mathcal{E}(i) & =\left[\begin{array}{l}
\mathcal{E}_{1}(i) \\
\mathcal{E}_{2}(i)
\end{array}\right]=\left[\begin{array}{l}
B^{T}(i) \\
B^{T}(i)
\end{array}\right]-\left[\begin{array}{c}
\tilde{B}^{\prime T}(i) \\
\tilde{B}^{\prime \prime T}(i)
\end{array}\right] \\
& =\left[\begin{array}{l}
B^{T}(i) \\
B^{T}(i)
\end{array}\right]-\left[\begin{array}{l}
\mathcal{H}_{1}(i) \\
\mathcal{H}_{2}(i)
\end{array}\right] \mathcal{X}(n) \\
& =\mathcal{B}(i)-\mathcal{H}(i) \mathcal{X}(n) .
\end{aligned}
$$

The optimal estimate of the tap-coefficient vector $\hat{\mathcal{X}}(n)$ is obtained by minimizing the cost function $J(n)$. Therefore, the optimal solution can be obtained by $\partial J(n) / \partial \mathcal{X}(n)=0$, i.e., see
(A.4), shown at the bottom of the page. Then, we can express the optimal estimate $\hat{\mathcal{X}}(n)$ as

$$
\hat{\mathcal{X}}(n)=\Phi^{-1}(n) \Theta(n)
$$

where, the correlation matrix $\Phi(n)$ and cross correlation $\Theta(n)$ are defined by

$$
\begin{aligned}
\Phi(n) & =\Phi_{1}(n)+\Phi_{2}(n) \\
\Theta(n) & =\Theta_{1}(n)+\Theta_{2}(n) \\
\Phi_{1}(n) & =\gamma_{1} \sum_{i=1}^{n} \lambda^{n-i} \mathcal{H}_{1}^{H}(i) \mathcal{H}_{1}(i) \\
\Phi_{2}(n) & =\gamma_{2} \sum_{i=1}^{n} \lambda^{n-i} \mathcal{H}_{2}^{H}(i) \mathcal{H}_{2}(i) \\
\Theta_{1}(n) & =\gamma_{1} \sum_{i=1}^{n} \lambda^{n-i} \mathcal{H}_{1}^{H}(i) B^{T}(i) \\
\Theta_{2}(n) & =\gamma_{2} \sum_{i=1}^{n} \lambda^{n-i} \mathcal{H}_{2}^{H}(i) B^{T}(i) .
\end{aligned}
$$

We can express the following recursive algorithms for updating the value of correlation matrix and cross correlation, respectively:

$$
\begin{aligned}
& \Phi(n)=\lambda \Phi(n-1)+\mathcal{H}^{H}(n)\left[\begin{array}{cc}
\gamma_{1} I & O \\
O & \gamma_{2} I
\end{array}\right] \cdot \mathcal{H}(n) \\
& \Theta(n)=\lambda \Theta(n-1)+\mathcal{H}^{H}(n)\left[\begin{array}{cc}
\gamma_{1} I & O \\
O & \gamma_{2} I
\end{array}\right] \cdot \mathcal{B}(n) .
\end{aligned}
$$

Lemma 1: Let $A, F, D$ be positive-definite matrices, related by $A=F^{-1}+C D^{-1} C^{H}$. The inverse matrix $A^{-1}$ can be expressed as follows: $A^{-1}=F-F C\left(D+C^{H} F C\right)^{-1} C^{H} F$.

With the correlation matrix $\Phi(n)$ assumed to be positively defined and therefore nonsingular, we may apply the above matrix inversion lemma to the recursive (A.12), i.e., we can let $A=\Phi(n), C=\mathcal{H}^{H}(n), F^{-1}=\lambda \Phi(n-1)$, and $D^{-1}=$ $\left[\begin{array}{cc}\gamma_{1} I & O \\ O & \gamma_{2} I\end{array}\right]$ to obtain the following recursive equation for the inversion of the correlation matrix:

$$
\begin{aligned}
\Phi^{-1}(n)= & \lambda^{-1} \Phi^{-1}(n-1)-\lambda^{-2} \Phi(n-1) \mathcal{H}^{H}(n) \\
& \cdot\left(\left[\begin{array}{cc}
\gamma_{1} I & O \\
O & \gamma_{2} I
\end{array}\right]^{-1}+\lambda^{-1} \mathcal{H}(n) \Phi^{-1}(n-1) \mathcal{H}^{H}(n)\right)^{-1} \\
& \cdot \mathcal{H}(n) \Phi^{-1}(n-1) .
\end{aligned}
$$

$$
\gamma_{1} \sum_{i=1}^{n} \lambda^{n-i} \mathcal{H}_{1}^{H}(i) B^{T}(i)-\gamma_{1} \sum_{i=1}^{n} \lambda^{n-i} \mathcal{H}_{1}^{H}(i) \mathcal{H}_{1}(i) \mathcal{X}(n)+\gamma_{2} \sum_{i=1}^{n} \lambda^{n-i} \mathcal{H}_{2}^{H}(i) B^{T}(i)-\gamma_{2} \sum_{i=1}^{n} \lambda^{n-i} \mathcal{H}_{2}^{H}(i) \mathcal{H}_{2}(i) \mathcal{X}(n)=0
$$


For convenience of computation, let

$$
\mathcal{P}(n)=\Phi^{-1}(n)
$$

and

$$
\begin{aligned}
\mathcal{K}(n)=\mathcal{P}(n-1) \mathcal{H}^{H}(n) & \left(\lambda\left[\begin{array}{cc}
\gamma_{1} I & O \\
O & \gamma_{2} I
\end{array}\right]^{-1}\right. \\
& \left.+\mathcal{H}(n) \mathcal{P}(n-1) \mathcal{H}^{H}(n)\right)^{-1}
\end{aligned}
$$

Using the definitions in (A.15) and (A.16), we may rewrite (A.14) as follows:

$$
\mathcal{P}(n)=\lambda^{-1}[I-\mathcal{K}(n) \mathcal{H}(n)] \mathcal{P}(n-1)
$$

We can arrange (A.16) and apply (A.17) to simplify (A.16) as follows:

$$
\begin{aligned}
\mathcal{K}(n)= & \lambda^{-1} \mathcal{P}(n-1) \mathcal{H}^{H}(n)\left[\begin{array}{cc}
\gamma_{1} I & O \\
O & \gamma_{2} I
\end{array}\right] \\
& -\lambda^{-1} \mathcal{K}(n) \mathcal{H}(n) \mathcal{P}(n-1) \mathcal{H}^{H}(n)\left[\begin{array}{cc}
\gamma_{1} I & O \\
O & \gamma_{2} I
\end{array}\right] \\
= & \lambda^{-1}[I-\mathcal{K}(n) \mathcal{H}(n)] \mathcal{P}(n-1) \mathcal{H}^{H}(n)\left[\begin{array}{cc}
\gamma_{1} I & O \\
O & \gamma_{2} I
\end{array}\right] \\
= & \mathcal{P}(n) \mathcal{H}^{H}(n)\left[\begin{array}{cc}
\gamma_{1} I & O \\
O & \gamma_{2} I
\end{array}\right] .
\end{aligned}
$$

Next, we wish to develop a recursive algorithm for updating the estimate $\hat{\mathcal{X}}(n)$. Thus, we express the estimate $\hat{\mathcal{X}}(n)$ at time $n$ as

$$
\begin{aligned}
\hat{\mathcal{X}}(n) & =\Phi^{-1}(n) \Theta(n)=\mathcal{P}(n) \Theta(n) \\
& =\mathcal{P}(n)\left(\lambda \Theta(n-1)+\mathcal{H}^{H}(n)\left[\begin{array}{cc}
\gamma_{1} I & O \\
O & \gamma_{2} I
\end{array}\right] \mathcal{B}(n)\right) \\
& =\lambda \mathcal{P}(n) \Theta(n-1)+\mathcal{P}(n) \mathcal{H}^{H}(n)\left[\begin{array}{cc}
\gamma_{1} I & O \\
O & \gamma_{2} I
\end{array}\right] \mathcal{B}(n) .
\end{aligned}
$$

Substituting (A.17) for $\mathcal{P}(n)$ in the first term in the right-hand side of (A.21), we get

$$
\begin{aligned}
\hat{\mathcal{X}}(n)= & {[I-\mathcal{K}(n) \mathcal{H}(n)] \mathcal{P}(n-1) \Theta(n-1) } \\
& +\mathcal{P}(n) \mathcal{H}^{H}(n)\left[\begin{array}{cc}
\gamma_{1} I & O \\
O & \gamma_{2} I
\end{array}\right] \mathcal{B}(n) \\
= & \hat{\mathcal{X}}-\mathcal{K}(n) \mathcal{H}(n) \hat{\mathcal{X}}(n-1) \\
& +\mathcal{P}(n) \mathcal{H}^{H}(n)\left[\begin{array}{cc}
\gamma_{1} I & O \\
O & \gamma_{2} I
\end{array}\right] \mathcal{B}(n) .
\end{aligned}
$$

Finally, using (A.18), we get the desired recursive algorithm for updating the estimated vector

$$
\hat{\mathcal{X}}(n)=\hat{\mathcal{X}}(n-1)+\mathcal{K}(n)[\mathcal{B}(n)-\mathcal{H}(n) \hat{\mathcal{X}}(n-1)]
$$

\section{ACKNOWLEDGMENT}

The authors would like to thank the anonymous reviewers and Associate Editor for their detailed comments and valuable suggestions.

\section{REFERENCES}

[1] M. B. Pursley, D. V. Sarwate, and W. E. Stark, "Error probability for direct-sequence spread-spectrum multiple-access communication, part I: Upper and lower bounds," IEEE Trans. Commun., vol. COM-30, pp. 975-984, May 1982.

[2] M. K. Simon, J. K. Omura, R. A. Scholtz, and B. K. Levitt, Spread Spectrum Communications. Rockville, MD: Computer Science Press, 1985.

[3] M. K. Varanasi and B. Aazhang, "Multistage detection in asynchronous code-division multiple-access communications," IEEE Trans. Commun., vol. 38, pp. 509-519, Apr. 1990.

[4] Z. Xie, R. T. Short, and C. K. Rushforth, "A family of suboptimum detectors for coherent multiuser communications," IEEE J. Select. Areas Commun., vol. 8, pp. 683-690, May 1990.

[5] A. Duel-Hallen, "Decorrelation decision-feedback multiuser detector for synchronous code-division multiple-access channel," IEEE Trans. Commun., vol. 41, pp. 285-290, Feb. 1993.

[6] J. Salz, "Digital transmission over cross-coupled linear channel," Bell Syst. Tech. J., vol. 64, pp. 1147-1159, July/Aug. 1985.

[7] A. Duel-Hallen, "Equalizers for multiple input/multiple output channels and PAM systems with cyclostationary input sequences," IEEE J. Select. Areas Commun., vol. 10, pp. 630-639, Apr. 1992.

[8] M. L. Honig, P. Crespo, and K. Steiglitz, "Suppression of near- and far-end crosstalk by linear pre- and post-filtering," IEEE J. Select. Areas Commun., vol. 10, pp. 614-629, Apr. 1992.

[9] R. Kohno, H. Imai, M. Hatori, and S. Pasupathy, "Combination of an adaptive array antenna and a canceller of interference for direct-sequence spread-spectrum multiple-access system," IEEE J. Select. Areas Commun., vol. 8, pp. 675-682, May 1990.

[10] A. J. Viterbi, "Very low rate convolution codes for maximum theoretical performance of spread spectrum multiple-access channels," IEEE J. Select. Areas Commun., vol. 8, pp. 641-649, May 1990.

[11] P. R. Patel and J. M. Holtzman, "Analysis of a simple successive interference cancellation scheme in a DS/CDMA system," IEEE J. Select. Areas Commun., vol. 12, pp. 796-807, June 1994.

[12] A. Radovic and B. Aazhang, "Iterative algorithms for joint data detection and delay estimation for code division multiple access communication systems," in Proc. Annu. Allerton Conf. Control and Computing Commun., Monticello, VA, Sept. 29-Oct. 11993.

[13] A. Kajieara and M. Nakagawa, "Crosscorrelation cancellation in SS/DS block demodulator," IEICE Trans., vol. E-74, pp. 2596-2602, Sept. 1991.

[14] A. Klein and P. W. Baier, "Linear unbiased data estimation in mobile radio systems applying CDMA," IEEE J. Select. Areas Commun., vol. 11, pp. 1058-1066, Sept. 1993.

[15] Z. Zvonar and D. Brady, "Multiuser detection in single-path fading channels," IEEE Trans. Commun., vol. 42, pp. 1729-1739, Feb./Mar./Apr. 1994.

[16] R. Lupas and S. Verdu, "Near-far resistance of multiuser detector in asynchronous channels," IEEE Trans. Commun., vol. 38, pp. 496-508, Apr. 1990.

[17] E. Eleftheriou and D. D. Falconer, "Tracking properties and steady-state performance of RLS adaptive filter algorithm," IEEE Trans. Acoust., Speech, Signal Processing, vol. ASSP-34, pp. 1097-1109, Oct. 1986.

[18] M. Abdulrahman and A. U. H. Sheikh, "Decision feedback equalization for CDMA in indoor wireless communications," IEEE J. Select. Areas Commun., vol. 12, pp. 698-706, May 1994.

[19] S. Moshavi, "Multi-user detection for DS-CDMA communications," IEEE Commun. Mag., pp. 124-136, Oct. 1996.

[20] P. N. Mongioudis, R. Tafazolli, and B. G. Evans, "LFSE interference cancellation in CDMA systems," in Proc. ICC'94, 1994, pp. 1160-1163.

[21] J. D. Laster and J. H. Reed, "Interference rejection in digital wireless communications," IEEE Signal Processing Mag., pp. 37-62, May 1997. 


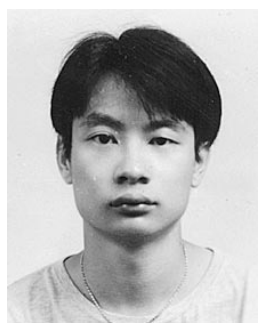

Wen-Sheng Hou was born in Taiwan, R.O.C., on November 8, 1967. He received the B.S. degree from the Department of Electrical Engineering, Tatung Institute of Technology, Taiwan, R.O.C., in 1991, and the M.S. degree from the Department of Electrical Engineering, National Tsing-Hua University, Hsin-Chu, Taiwan, in 1993. He is currently working toward the Ph.D. degree in electrical engineering at National Tsing-Hua University.

His research interests include signal processing and communication.

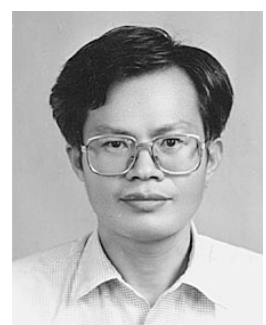

Bor-Sen Chen (M'82-SM'89) received the B.S. degree from Tatung Institute of Technology, Taiwan, R.O.C., in 1970, the M.S. degree from National Central University, Taiwan, R.O.C., in 1973, and the Ph.D. degree from the Univeraity of Southern California, Los Angeles, in 1982.

He was a Lecturer, Associate Professor, and Professor at Tatung Institute of Technology from 1973 to 1987 . He is now a Professor at National Tsing-Hua University, Hsin-Chu, Taiwan, R.O.C. His current research interests include control signal processing and communication.

Dr. Chen has received the Distinguished Research Award from the National Science Council of Taiwan. He now holds a Chair of the Foundation of Outstanding Scholars. 